\title{
Interactive comment on "A complete glacier inventory of the Antarctic Peninsula based on Landsat7 images from 2000-2002 and other pre-existing datasets" by Jacqueline Huber et al.
}

\section{B. Marzeion (Referee) \\ ben.marzeion@uni-bremen.de}

Received and published: 19 October 2016

Huber et al. present a complete and comprehensive inventory of glaciers on the Antarctic Peninsula, based on the aggregation of a diverse set of previously published data. The availability of such data sets is a prerequisite for understanding, and quantifying, the sensitivity and response of glaciers to climate variability and change, including all the consequences (in the case of the AP, primarily sea-level rise). A data set as presented here is long-needed for the AP, is a great step towards completion of such inventories on the global scale and will be used in many future studies. The manuscript is generally well written (see below for a few specific comments), and the authors describe the workflow well and understandable also for non-specialist 
readers. However, before I can recommend the manuscript for publication, one general (and somewhat major) and several specific (and mostly minor) comments should be addressed. Please see below for the details.

\section{General comments:}

- I see only one issue with the manuscript that will require some more substantial work to address: the handling (or lack of handling) of uncertainties. The authors address uncertainties in section 6.3. However, they do not attempt to translate the discussion here into uncertainties regarding their results - e.g., the hard-todetermine grounding line (Sect. 6.3.3) has a strong influence on the glacier area: how does that translate into uncertainty of the total area number for the AP given in the abstract and elsewhere?

Addressing this properly, i.e. trying to come up with uncertainty estimates for the numbers given in the abstract, the main text, and Table 3 , will also require a reorganization of the manuscript. What is now in Sect. 6.3 would have to be discussed (together with the derivation of the uncertainty estimates) before the results section (Sect. 5).

It may not be possible to estimate the uncertainties for all the derived variables, and for some where it is possible, it may only be very indicative (e.g., is the error in the $1 \%$ range or in the $10 \%$ range). But in the current shape, the manuscript creates the (wrong, I'm sure) impression of blindness of the authors towards this issue - also because of the way many numbers are presented (e.g., mean thickness including digits on the $\mathrm{mm}$ scale, or the total area of glaciers on the AP to the $0.1 \mathrm{~km} 2$ scale).

\section{Specific comments:}

- L20ff: all the numbers given in the abstract should include an uncertainty estimate 
- see above.

- L26ff: since you do not discuss the ELA in the manuscript, having point (1) in the abstract is a bit of a surprise without additional information, I would recommend removing it; points (2) and (3) could be combined into one statement on the sensitivity of the region to marine-induced ice dynamic effects.

Interactive

comment

- L39/Fig. 1: the first reference to Fig. 1 comes before the definition of the abbreviation LIMA - so this definition should perhaps be in the caption of Fig. 1.

- L65: "the part of the AP north of 70 S represents..."

- L78: "experience" is typeset is a smaller font?

- L79: delete "help".

- Sect. 3: it would help in some places to change the formulation to better separate what was done by the authors of this manuscript from what was done by the authors of the references. E.g., instead of writing ".. groundling lines ... were used, modified in places..." (L119) an active voice clarifies: "... they used grounding lines and modified them in places..."; or instead of "Other sources ... were used" (L164): "They used other sources...".

- L107: it would be good if you indicate (one sentence) how Cook et al. (2012) deal with these gaps.

- L188: "'...glaciers on Greenland with a strong dynamic connection the Greenland ice sheet should..."

- L190: "strong connection" - I think "strong" is ambiguous here (especially in connection with the word "strong" in the previous sentence. As it refers to space here, perhaps "broad" or "extended" would be better? 
- L207ff: I think the greater problem (still not very big...) than the spatial inconsistency is that the thickness estimation also depends on velocity, which is of course connected to ice divides, outcrops, etc. That is, there is a certain degree of internal inconsistency among the data sets used. This could only be fixed by repeating (perhaps even iteratively) certain parts of the analyses that lead to the data sets used - this would obviously be too much to ask for, but I think this problem needs to be mentioned in the manuscript, probably best in the discussion section.

- L219ff: can be shortened to "The sea level equivalent (SLE) of the ice volume is calculated by assuming a mean ice density of $900 \mathrm{~kg} \mathrm{~m}-3$ and dividing it by the ocean surface area (3.625 × 108 km2; Cogley, 2012)."

- L225: isostatic effects (increasing ocean volume and lifting the land surface) probably have a bigger effect than the dilution, cooling, and ocean dynamics.

- L230: "100 m bins"

- L238f: isn't this the same as stating "the rock outcrops cover an area of 1709 km2"? - it would be simpler.

- L240: giving the area of the smallest glacier in the data set has limited value, as it is the smallest "detected" glacier, and there are presumably a large number of very small glaciers missing in the data set (see the discussion in Pfeffer et al., 2014, around that). It would be more helpful to refer to the histogram (Fig. 4).

- Table 2: remove digits in the numbers that are clearly within the uncertainty range (e.g., giving minimum elevation on a $\mathrm{mm}$ scale...).

- L247: "a csv-file".

- L259/Fig. 4: please specify the size classes (either in a small table, or perhaps better, on the figure axis). 
- L268: “...being $300 \mathrm{~m}$ or more higher than ...”

- L269: what does "in the south" mean here?

- Tab. 3: it would be good to have uncertainty estimates also here.

- Fig. 6 (also Fig. S3): please indicate the percentiles corresponding to the box and whiskers in the caption.

- L399: "For instance, for a glacier with missing values at the coast, the..."

- L405f: this would also help removing internal inconsistencies, see above.

- L435: “...of a land-terminated glacier...”.

- L459: repetition from the paragraph above.

- L470f: "The lowest $800 \mathrm{~m}$ and hence $50 \%$ of the glacierized area are prone to rising ablation and mass loss,..." I don't understand where the number $800 \mathrm{~m}$ comes from?

- L238/L481: excluding islands $<0.5 \mathrm{~km} 2$ or $0.05 \mathrm{~km} 2$ ?

Interactive comment on Earth Syst. Sci. Data Discuss., doi:10.5194/essd-2016-47, 2016. 